\title{
Almost Periodic Time Scales and Almost Periodic Functions on Time Scales
}

\author{
Yongkun Li and Bing Li \\ Department of Mathematics, Yunnan University, Kunming, Yunnan 650091, China \\ Correspondence should be addressed to Yongkun Li; yklie@ynu.edu.cn
}

Received 24 October 2014; Revised 13 December 2014; Accepted 15 December 2014

Academic Editor: Allan C. Peterson

Copyright (C) 2015 Y. Li and B. Li. This is an open access article distributed under the Creative Commons Attribution License, which permits unrestricted use, distribution, and reproduction in any medium, provided the original work is properly cited.

We propose some new concepts of almost periodic time scales and almost periodic functions on time scales and give some basic properties of these new types of almost periodic time scales and almost periodic functions on time scales. We also give some comments on a recent paper by Wang and Agarwal (2014) concerning a new almost periodic time scale.

\section{Introduction}

The theory of almost periodic functions was introduced in the literature around 1924-1926 by Bohr [1]. The notion of almost periodicity, which generalizes the concept of periodicity, plays a crucial role in various fields including harmonic analysis, physics, and dynamical systems. On the other hand, the theory of time scales, which was introduced by Hilger [2] in order to unify continuous and discrete analysis, has recently received lots of attention. The field of dynamic equations on time scale contains links and extends the classical theory of differential and difference equations.

In order to study the almost periodic dynamic equations on time scales, a concept of almost periodic time scales was proposed in [3]. Based on this concept, almost periodic functions [3], pseudo almost periodic functions [4], almost automorphic functions [5], weighted pseudo almost automorphic functions [6], and weighted piecewise pseudo almost automorphic functions [7] on time scales were defined successively. Also, some works have been done under the concept of almost periodic time scales (see [8-11]). Although the concept of almost periodic time scales in [3] can unify the the continuous and discrete situations effectively, it is very restrictive in the sense that it only comprises of time scale, $\mathbb{T}$, for which the graininess function, $\mu(t)$, is constant (or piecewise constant). This excludes many interesting time scales. Therefore, it is a challenging and important problem in theories and applications to find a new concept of periodic time scales.

Recently, Wang and Agarwal [12] have constructed an almost periodic time scale and given a redefined concept of almost periodic functions under the sense of this timescale concept. We argue that the definition of this almost periodic time scale contains some vagueness. In order to remove the vagueness, our main purpose of this paper is to propose some new concepts of almost periodic time scales and almost periodic functions on time scales and give some basic properties of these new types of almost periodic time scales and almost periodic functions on time scales. Based on these new types of almost periodic time scales and almost periodic functions on time scales, one can further study pseudo almost periodic functions, almost automorphic functions, weighted pseudo almost periodic functions, and weighted pseudo almost automorphic functions on time scales and the existence problems of these functional classes solutions to dynamic equations on time scales; in particular, one can study the existence of almost periodic solutions to population models, neural networks on time scales and so on.

\section{Preliminaries}

In this section, we shall first recall some basic definitions which are used in what follows.

Let $\mathbb{T}$ be a nonempty closed subset (time scale) of $\mathbb{R}$. The forward and backward jump operators $\sigma, \rho: \mathbb{T} \rightarrow \mathbb{T}$ and the graininess $\mu: \mathbb{T} \rightarrow \mathbb{R}^{+}$are defined, respectively, by

$$
\begin{gathered}
\sigma(t)=\inf \{s \in \mathbb{T}: s>t\}, \quad \rho(t)=\sup \{s \in \mathbb{T}: s<t\}, \\
\mu(t)=\sigma(t)-t .
\end{gathered}
$$


A point $t \in \mathbb{T}$ is called left-dense if $t>\inf \mathbb{T}$ and $\rho(t)=t$, left-scattered if $\rho(t)<t$, right-dense if $t<\sup \mathbb{\square}$ and $\sigma(t)=t$, and right-scattered if $\sigma(t)>t$.

A function $f: \mathbb{T} \rightarrow \mathbb{R}$ is right-dense continuous provided it is continuous at right-dense point in $\mathbb{T}$ and its leftside limits exist at left-dense points in $\mathbb{T}$. If $f$ is continuous at each right-dense point and each left-dense point, then $f$ is said to be a continuous function on $\mathbb{T}$.

For more knowledge of time scales, one can refer to [13].

Throughout this paper, $\mathbb{E}^{n}$ denotes $\mathbb{R}^{n}$ or $\mathbb{C}^{n}, D$ denotes an open set in $\mathbb{E}^{n}$ or $D=\mathbb{E}^{n}, S$ denotes an arbitrary compact subset of $D$.

Definition 1 (see [14]). A subset $S$ of $\mathbb{R}$ is called relatively dense if there exists a positive number $L$ such that $[a, a+L] \cap$ $S \neq \phi$ for all $a \in \mathbb{R}$. The number $L$ is called the inclusion length.

Definition 2 (see [3]). A time scale $\mathbb{\square}$ is called an almost periodic time scale if

$$
\Pi:=\{\tau \in \mathbb{R}: t \pm \tau \in \mathbb{T}, \forall t \in \mathbb{T}\} \neq\{0\} .
$$

Definition 3 (see [3]). Let $\mathbb{T}$ be an almost periodic time scale. A function $f \in C\left(\mathbb{T} \times D, \mathbb{E}^{n}\right)$ is called an almost periodic function in $t \in \mathbb{T}$ uniformly for $x \in D$ if the $\varepsilon$-translation set of $f$

$$
\begin{gathered}
E\{\varepsilon, f, S\}=\{\tau \in \Pi:|f(t+\tau, x)-f(t, x)|<\varepsilon, \\
\forall(t, x) \in \mathbb{T} \times S\}
\end{gathered}
$$

is a relatively dense set in $\mathbb{T}$ for all $\varepsilon>0$ and for each compact subset $S$ of $D$; that is, for any given $\varepsilon>0$ and each compact subset $S$ of $D$, there exists a constant $l(\varepsilon, S)>0$ such that each interval of length $l(\varepsilon, S)$ contains a $\tau(\varepsilon, S) \in E\{\varepsilon, f, S\}$ such that

$$
|f(t+\tau, x)-f(t, x)|<\varepsilon, \quad \forall(t, x) \in \mathbb{T} \times S .
$$

$\tau$ is called the $\varepsilon$-translation number of $f$ and and $l(\varepsilon, S)$ is called the inclusion length of $E\{\varepsilon, f, S\}$.

Remark 4. Definition 3 requires that the inequality (4) holds for all $(t, x) \in \mathbb{T} \times S$. It is a very restrictive condition.

Let $\tau$ be a number; set the time scales:

$$
\begin{gathered}
\mathbb{T}:=\bigcup_{i=-\infty}^{+\infty}\left[\alpha_{i}, \beta_{i}\right], \\
\mathbb{T}^{\tau}:=\mathbb{T}+\tau=\{t+\tau: \forall t \in \mathbb{T}\}:=\bigcup_{i=-\infty}^{+\infty}\left[\alpha_{i}^{\tau}, \beta_{i}^{\tau}\right] .
\end{gathered}
$$

Authors of [12] defined the distance between two time scales $\mathbb{T}$ and $\mathbb{T}^{\tau}$ by

$$
d\left(\mathbb{T}, \mathbb{T}^{\tau}\right)=\max \left\{\sup _{i \in \mathbb{Z}}\left|\alpha_{i}-\alpha_{i}^{\tau}\right|, \sup _{i \in \mathbb{Z}}\left|\beta_{i}-\beta_{i}^{\tau}\right|\right\} .
$$

Then, they gave the following two definitions.
Definition 5 (see [12]). We say $\mathbb{T}$ is an almost periodic time scale if, for any given $\varepsilon>0$, there exists a constant $l(\varepsilon)>0$ such that each interval of length $l(\varepsilon)$ contains a $\tau(\varepsilon)$ such that

$$
d\left(\mathbb{T}, \mathbb{T}^{\tau}\right)<\varepsilon
$$

that is, for any $\varepsilon>0$, the following set

$$
E(\mathbb{T}, \varepsilon)=\left\{\tau \in \mathbb{R}, d\left(\mathbb{T}, \mathbb{T}^{\tau}\right)<\varepsilon\right\}
$$

is relatively dense.

Definition 6 (see [12]). Let $\mathbb{T}$ be an almost periodic time scale. A function $f \in C\left(\mathbb{T} \times D, \mathbb{E}^{n}\right)$ is called an almost periodic function in $t \in \mathbb{Z}$ uniformly for $x \in D$ if the $\varepsilon$-translation set of $f$

$$
\begin{gathered}
E\{\varepsilon, f, S\}=\{\tau:|f(t+\tau, x)-f(t, x)|<\varepsilon, \\
\left.\forall(t, x) \in\left(\mathbb{T} \cap \mathbb{T}^{-\tau}\right) \times S\right\}
\end{gathered}
$$

is a relatively dense set for all $\varepsilon>0$ and for each compact subset $S$ of $D$; that is, for any given $\varepsilon>0$ and each compact subset $S$ of $D$, there exists a constant $l(\varepsilon, S)>0$ such that each interval of length $l(\varepsilon, S)$ contains a $\tau(\varepsilon, S) \in E\{\varepsilon, f, S\}$ such that

$$
|f(t+\tau, x)-f(t, x)|<\varepsilon, \quad \forall(t, x) \in\left(\mathbb{T} \cap \mathbb{T}^{-\tau}\right) \times S .
$$

$\tau$ is called the $\varepsilon$-translation number of $f$ and and $l(\varepsilon, S)$ is called the inclusion length of $E\{\varepsilon, f, S\}$.

We comment that

(i) Definition 5 is very vague because one does not know how to calculate (6). For example, what are the $\alpha_{i}^{\tau}, \beta_{i}^{\tau}$ ? Since if the $i$ in $\alpha_{i}$ and the $i$ in $\alpha_{i}^{\tau}$ are the same, then

$$
\alpha_{i}^{\tau}=\alpha_{i}+\tau, \quad \beta_{i}^{\tau}=\beta_{i}+\tau ;
$$

according to (6), we have

$$
d\left(\mathbb{T}, \mathbb{T}^{\tau}\right)=\tau .
$$

In this case, by Definition 5, the set

$$
E(\mathbb{T}, \varepsilon)=\left\{\tau \in \mathbb{R}, d\left(\mathbb{T}, \mathbb{T}^{\tau}\right)<\varepsilon\right\}
$$

can not be relatively dense. If the $i$ in $\alpha_{i}$ and the $i$ in $\alpha_{i}^{\tau}$ are different, what is the relation between these two $i$ 's?

(ii) Definition 6 is not well defined because $\mathbb{T} \cap \mathbb{T}^{-\tau}$ may be an empty set. For example, if we take $\mathbb{T}=\mathbb{Z}$ and $\tau=r, r \in(0,1)$, then $\mathbb{T} \cap \mathbb{T}^{-\tau}=\emptyset$. But $E(\mathbb{T}, \varepsilon)=$ $\left\{\tau \in \mathbb{R}, d\left(\mathbb{T}, \mathbb{T}^{\tau}\right)<\varepsilon\right\}$ is relatively dense since for every $\varepsilon>0$, there exists a $\tau=k+r(\varepsilon) \in E(\mathbb{T}, \varepsilon)$, where $k \in \mathbb{Z}, r(\varepsilon) \in(0,1)$ with $r(\varepsilon)<\varepsilon$.

\section{Almost Periodic Time Scales}

In order to remove the vagueness of Definition 5 and rule out the situation that $\mathbb{\mathbb { N }} \cap \mathbb{\mathbb { T }}^{-\tau}$ may be empty set in Definition 5 , we give the following definitions. 
Definition 7 . Let $\mathbb{T}_{1}$ and $\mathbb{T}_{2}$ be two time scales, we define

$$
\operatorname{dist}\left(\mathbb{T}_{1}, \mathbb{T}_{2}\right)=\max \left\{\sup _{t \in \mathbb{T}_{1}}\left\{\operatorname{dist}\left(t, \mathbb{T}_{2}\right)\right\}, \sup _{t \in \mathbb{\mathbb { T }}_{2}}\left\{\operatorname{dist}\left(t, \mathbb{\mathbb { T }}_{1}\right)\right\}\right\} \text {, }
$$

where $\operatorname{dist}\left(t, \mathbb{T}_{2}\right)=\inf _{s \in \mathbb{T}_{2}}\{|t-s|\}$ and $\operatorname{dist}\left(t, \mathbb{T}_{1}\right)=\inf _{s \in \mathbb{T}_{1}}\{\mid t-$ $s \mid\}$. Let $\tau \in \mathbb{R}$ and $\mathbb{T}$ be a time scale; we define

$$
\operatorname{dist}\left(\mathbb{T}, \mathbb{\mathbb { T }}_{\tau}\right)=\max \left\{\sup _{t \in \mathbb{\mathbb { T }}}\left\{\operatorname{dist}\left(t, \mathbb{\mathbb { T }}_{\tau}\right)\right\}, \sup _{t \in \mathbb{T}_{\tau}}\{\operatorname{dist}(t, \mathbb{T})\}\right\},
$$

where $\mathbb{T}_{\tau}:=\mathbb{T} \cap\{\mathbb{T}-\tau\}=\{t-\tau: \forall t \in \mathbb{T}\}$, dist $\left(t, \mathbb{T}_{\tau}\right)=$ $\inf _{s \in \mathbb{T}_{\tau}}\{|t-s|\}$, and $\operatorname{dist}(t, \mathbb{T})=\inf _{s \in \mathbb{T}}\{|t-s|\}$.

From Definition 7 and noticing that time scales are closed subsets of $\mathbb{R}$, we have the following.

Lemma 8. Let $\mathbb{T}_{1}$ and $\mathbb{T}_{2}$ be two time scales; then $\operatorname{dist}\left(\mathbb{T}_{1}, \mathbb{T}_{2}\right)=$ 0 if and only if $\mathbb{T}_{1}=\mathbb{T}_{2}$.

Definition 9. A time scale $\mathbb{T}$ is called an almost periodic time scale if for every $\varepsilon>0$, there exists a constant $l(\varepsilon)>0$ such that each interval of length $l(\varepsilon)$ contains a $\tau(\varepsilon)$ such that $\mathbb{T}_{\tau} \neq$ $\emptyset$ and

$$
\operatorname{dist}\left(\mathbb{T}, \mathbb{T}_{\tau}\right)<\varepsilon
$$

that is, for any $\varepsilon>0$, the following set

$$
\Pi(\mathbb{T}, \varepsilon)=\left\{\tau \in \mathbb{R}, \operatorname{dist}\left(\mathbb{T}, \mathbb{\mathbb { T }}_{\tau}\right)<\varepsilon\right\}
$$

is relatively dense. $\tau$ is called the $\varepsilon$-translation number of $\mathbb{T}$ and $l(\varepsilon)$ is called the inclusion length of $\Pi(\mathbb{T}, \varepsilon)$.

Obviously, if $\mathbb{T}$ is an almost periodic time scale, then inf $\mathbb{T}=-\infty$ and sup $\mathbb{T}=+\infty$, if $\mathbb{T}$ is a periodic time scale (see [15]), then $\operatorname{dist}\left(\mathbb{\mathbb { T }}, \mathbb{T}_{\tau}\right)=0$; that is, $\mathbb{T}=\mathbb{T}_{\tau}$.

Lemma 10. Let $\mathbb{T}$ be an almost periodic time scale; then

(i) if $\tau \in \Pi(\mathbb{T}, \varepsilon)$, then $t+\tau \in \mathbb{T}$ for all $t \in \mathbb{T}_{\tau}$;

(ii) if $\varepsilon_{1}<\varepsilon_{2}$, then $\Pi\left(\mathbb{T}, \varepsilon_{1}\right) \subset \Pi\left(\mathbb{T}, \varepsilon_{2}\right)$;

(iii) if $\tau \in \Pi(\mathbb{T}, \varepsilon)$, then $-\tau \in \Pi(\mathbb{T}, \varepsilon)$ and $\operatorname{dist}\left(\mathbb{T}_{\tau}, \mathbb{T}\right)=$ $\operatorname{dist}\left(\mathbb{T}_{-\tau}, \mathbb{T}\right)$;

(iv) if $\tau_{1}, \tau_{2} \in \Pi(\mathbb{T}, \varepsilon)$, then $\tau_{1}+\tau_{2} \in \Pi(\mathbb{T}, 2 \varepsilon)$.

Proof. (i), (ii), and (iii) are trivial; we only prove (iv). If $\tau_{1}, \tau_{2} \in$ $\Pi(\mathbb{T}, \varepsilon)$, then

$$
\operatorname{dist}\left(\mathbb{T}_{\tau_{1}+\tau_{2}}, \mathbb{T}\right) \leq \operatorname{dist}\left(\mathbb{T}_{\tau_{1}}, \mathbb{\mathbb { T }}\right)+\operatorname{dist}\left(\mathbb{T}_{-\tau_{2}}, \mathbb{\mathbb { v }}\right)<2 \text {; }
$$

that is, $\tau_{1}+\tau_{2} \in \Pi(\mathbb{T}, 2 \varepsilon)$. The proof is complete.

Lemma 11. Let $\mathbb{T}$ be an almost periodic time scale; then, there exists a sequence $\left\{\tau_{n}\right\} \subset \Pi(\mathbb{T}, \varepsilon)$ such that $\operatorname{dist}\left(\mathbb{T}, \mathbb{T}_{\tau_{n}}\right) \rightarrow 0$ as $n \rightarrow \infty$. Hence, $\overline{\bigcup_{n=0}^{\infty} \mathbb{T}_{\tau_{n}}}=\mathbb{T}$.
Proof. For each $n=1,2, \ldots$, take $\tau_{n} \in \Pi(\mathbb{T}, \varepsilon / n)$; then,

$$
\operatorname{dist}\left(\mathbb{T}, \mathbb{T}_{\tau_{n}}\right)<\frac{\varepsilon}{n}, \quad n=1,2, \ldots
$$

The proof is complete.

For convenience, we introduce some notations. Let $\alpha=$ $\left\{\alpha_{n}\right\}$ and $\beta=\left\{\beta_{n}\right\}$ be two sequences. Then, $\beta \subset \alpha$ means that $\beta$ is a subsequence of $\alpha ; \alpha+\beta=\left\{\alpha_{n}+\beta_{n}\right\} ;-\alpha=\left\{-\alpha_{n}\right\}$; and $\alpha$ and $\beta$ are common subsequences of $\alpha^{\prime}$ and $\beta^{\prime}$, respectively, meaning that $\alpha_{n}=\alpha_{n(k)}^{\prime}$ and $\beta_{n}=\beta_{n(k)}^{\prime}$ for some given function $n(k)$.

Theorem 12. Let $\mathbb{T}$ be an almost periodic time scale; then, for any given sequence $\alpha^{\prime} \subset \Pi(\mathbb{T}, \varepsilon)$, there exist a subsequence $\beta \subset$ $\alpha^{\prime}$ such that $\mathbb{T}_{\beta_{n}}$ converges to some time scale $\mathbb{T}^{*}$; that is, for any given $\varepsilon>0$, there exists $N>0$ such that $n>N$ implies $\operatorname{dist}\left(\mathbb{T}_{\beta_{n}}, \mathbb{T}^{*}\right)<\varepsilon$. Furthermore, $\mathbb{T}^{*}$ is also an almost periodic time scale.

Proof. For any $\varepsilon>0$, let $l=l(\varepsilon / 4)$ be an inclusion length of $\Pi(\mathbb{T}, \varepsilon / 4)$. For any given sequence $\alpha^{\prime}=\left\{\alpha_{n}^{\prime}\right\}$, we denote $\alpha_{n}^{\prime}=\tau_{n}^{\prime}+\gamma_{n}^{\prime}$, where $\tau_{n}^{\prime} \in \Pi(\mathbb{T}, \varepsilon / 4), \gamma_{n}^{\prime} \in \Pi$, and $0 \leq \gamma_{n}^{\prime} \leq l$, $n=1,2, \ldots$. Therefore, there exists a subsequence $\gamma=\left\{\gamma_{n}\right\} \subset$ $\gamma^{\prime}=\left\{\gamma_{n}^{\prime}\right\}$ such that $\gamma_{n} \rightarrow s$ as $n \rightarrow \infty, 0 \leq s \leq l$.

Also, it follows from Definition 9 that there exists $\delta(\varepsilon)>0$ such that if $t_{1}, t_{2} \in \Pi(\mathbb{T}, \varepsilon)$ and $\left|t_{1}-t_{2}\right|<\delta$; then,

$$
\operatorname{dist}\left(\mathbb{T}_{t_{1}}, \mathbb{T}_{t_{2}}\right)<\frac{\varepsilon}{2}
$$

Since $\gamma$ is a convergent sequence, there exists $N=N(\delta)$ so that $p, m \geq N$ implies $\left|\gamma_{p}-\gamma_{m}\right|<\delta$. Now, one can take $\alpha \subset \alpha^{\prime}$, $\tau \subset \tau^{\prime}=\left\{\tau_{n}^{\prime}\right\}$ such that $\alpha, \tau$ common with $\gamma$, then for any integers $p, m \geq N$, we have

$$
\begin{aligned}
\operatorname{dist}\left(\mathbb{T}_{\tau_{p}-\tau_{m}}, \mathbb{T}\right) & \leq \operatorname{dist}\left(\mathbb{T}_{\tau_{p}-\tau_{m}}, \mathbb{T}_{\tau_{m}}\right)+\operatorname{dist}\left(\mathbb{T}_{\tau_{m}}, \mathbb{T}\right) \\
& <\frac{\varepsilon}{4}+\frac{\varepsilon}{4}=\frac{\varepsilon}{2} ;
\end{aligned}
$$

that is

$$
\left(\alpha_{p}-\alpha_{m}\right)-\left(\gamma_{p}-\gamma_{m}\right)=\tau_{p}-\tau_{m} \in \Pi\left(\mathbb{T}, \frac{\varepsilon}{2}\right) .
$$

Hence, we can obtain

$$
\begin{aligned}
\operatorname{dist}\left(\mathbb{T}_{\alpha_{p}}, \mathbb{T}_{\alpha_{m}}\right) & \leq \operatorname{dist}\left(\mathbb{T}_{\alpha_{p}-\alpha_{m}}, \mathbb{T}\right) \\
& \leq \operatorname{dist}\left(\mathbb{T}_{\alpha_{p}-\alpha_{m}}, \mathbb{T}_{\gamma_{p}-\gamma_{m}}\right)+\operatorname{dist}\left(\mathbb{T}_{\gamma_{p}-\gamma_{m}}, \mathbb{T}\right) \\
& <\frac{\varepsilon}{2}+\frac{\varepsilon}{2}=\varepsilon .
\end{aligned}
$$

Thus, we can take sequences $\alpha^{(k)}=\left\{\alpha_{n}^{(k)}\right\}, k=1,2, \ldots$, and $\alpha^{(k+1)} \subset \alpha^{(k)} \subset \alpha$ such that for any integers $m$, $p$, the following holds:

$$
\operatorname{dist}\left(\mathbb{T}_{\alpha_{p}^{(k)}}, \mathbb{T}_{\alpha_{m}^{(k)}}\right)<\frac{1}{k}, \quad k=1,2, \ldots
$$


For all sequences $\alpha^{(k)}, k=1,2, \ldots$, we can take a sequence $\beta=\left\{\beta_{n}\right\}, \beta_{n}=\alpha_{n}^{(n)}$, then it is easy to see that $\left\{T_{\beta_{n}}\right\} \subset\left\{T_{\alpha_{n}}\right\}$ for any integers $p, m$ with $p<m$; the following holds:

$$
\operatorname{dist}\left(\mathbb{T}_{\beta_{p}}, \mathbb{T}_{\beta_{m}}\right)<\frac{1}{p} \text {. }
$$

Therefore, $\left\{\mathbb{T}_{\beta_{n}}\right\}$ converges to some $\mathbb{T}^{*}$, where $\beta=\left\{\beta_{n}\right\} \subset \alpha$, which is a closed subset of $\mathbb{R}$, that is, $\operatorname{dist}\left(\mathbb{T}_{\beta_{n}}, \mathbb{T}^{*}\right) \rightarrow 0$ as $n \rightarrow \infty$.

Finally, for any given $\varepsilon>0$, one can take $\tau \in \Pi(\mathbb{T}, \varepsilon)$; then, the following holds:

$$
\operatorname{dist}\left(\mathbb{T}_{\beta_{n}+\tau}, \mathbb{T}_{\beta_{n}}\right)<\varepsilon .
$$

Let $n \rightarrow \infty$; we have

$$
\operatorname{dist}\left(\mathbb{T}_{\tau}^{*}, \mathbb{T}^{*}\right)<\varepsilon,
$$

which implies that $\Pi\left(\mathbb{T}^{*}, \varepsilon\right)$ is relatively dense. Therefore, $\mathbb{T}^{*}$ is an almost periodic time scale. This completes the proof.

Theorem 13. Let $\mathbb{T}$ be a time scale; if for any sequence $\alpha^{\prime} \subset$ $\Pi(\mathbb{T}, \varepsilon)$, there exists $\alpha \subset \alpha^{\prime}$ such that every $\mathbb{T}_{\alpha_{n}} \neq \emptyset$ and $\left\{\mathbb{T}_{\alpha_{n}}\right\}$ converges to a time scale $\mathbb{T}^{*}$; then, $\mathbb{T}$ is also an almost periodic time scale.

Proof. For contradiction, if this is not true, then there exists $\varepsilon_{0}>0$ such that for any sufficiently large $l>0$, we can find an interval with length of $l$ and there is no $\varepsilon_{0}$-translation numbers of $\mathbb{T}$ in this interval; that is, every point in this interval is not in $\Pi\left(\mathbb{T}, \varepsilon_{0}\right)$.

One can take a number $\alpha_{1}^{\prime} \in \Pi\left(\mathbb{T}, \varepsilon_{0}\right)$ and find an interval $\left(a_{1}, b_{1}\right)$ with $b_{1}-a_{1}>2\left|\alpha_{1}^{\prime}\right|$, where $a_{1}, b_{1} \in \Pi\left(\mathbb{T}, \varepsilon_{0}\right)$ such that there is no $\varepsilon_{0}$-translation numbers of $f(t, x)$ in this interval. Next, taking $\alpha_{2}^{\prime}=(1 / 2)\left(a_{1}+b_{1}\right)$, obviously, $\alpha_{2}^{\prime}-\alpha_{1}^{\prime} \in\left(a_{1}, b_{1}\right)$, so $\alpha_{2}^{\prime}-\alpha_{1}^{\prime} \notin \Pi\left(T, \varepsilon_{0}\right)$; then, one can find an interval $\left(a_{2}, b_{2}\right)$ with $b_{2}-a_{2}>2\left(\left|\alpha_{1}^{\prime}\right|+\left|\alpha_{2}^{\prime}\right|\right)$, where $a_{2}, b_{2} \in \Pi\left(\mathbb{T}, \varepsilon_{0}\right)$ such that there are no $\varepsilon_{0}$-translation numbers of $f(t, x)$ in this interval. Next, taking $\alpha_{3}^{\prime}=(1 / 2)\left(a_{2}+b_{2}\right)$, obviously, $\alpha_{3}^{\prime}-\alpha_{2}^{\prime}, \alpha_{3}^{\prime}-\alpha_{1}^{\prime} \notin \Pi\left(\mathbb{T}, \varepsilon_{0}\right)$. One can repeat these processes again and again; one can find $\alpha_{4}^{\prime}, \alpha_{5}^{\prime}, \ldots$, such that $\alpha_{i}^{\prime}-\alpha_{j}^{\prime} \notin \Pi\left(\mathbb{T}, \varepsilon_{0}\right), i>j$. Hence, for any $i \neq j, i, j=1,2, \ldots$, without loss of generality; letting $i>j$, we have

$$
\operatorname{dist}\left(\mathbb{T}_{\alpha_{i}^{\prime}}, \mathbb{T}_{\alpha_{j}^{\prime}}\right)=\operatorname{dist}\left(\mathbb{T}_{\alpha_{i}^{\prime}-\alpha_{j}^{\prime}}, \mathbb{T}\right) \geq \varepsilon_{0} .
$$

Therefore, there is no convergent subsequence of $\left\{\mathbb{T}_{\alpha_{n}^{\prime}}\right\}$; this is a contradiction. Thus, $\mathbb{T}$ is almost periodic time scale. This completes the proof.

Theorem 14. Let $\mathbb{T}$ be an almost periodic time scale; then, for any $\tau(\varepsilon) \in \Pi(\mathbb{T}, \varepsilon)$, there exists a constant $l(\varepsilon)>0$ such that each interval of length $l(\varepsilon)$ contains a $\tau(\varepsilon) \in \Pi(\mathbb{T}, \varepsilon)$ such that

$$
|\sigma(t+\tau)-\sigma(t)-\tau|<\varepsilon, \quad \forall t \in \mathbb{T}_{\tau} .
$$

Proof. For any $\tau \in \Pi(\mathbb{T}, \varepsilon)$ and $t \in \mathbb{T}_{\tau}$, if $t$ is right dense, then there exist $t_{n} \in \mathbb{T}$ such that

$$
\lim _{n \rightarrow \infty} t_{n}=t, \quad t_{n}>t .
$$

If there exists a subsequence $\left\{t_{n_{k}}\right\}$ of $\left\{t_{n}\right\}$ such that $\left\{t_{n_{k}}\right\} \subset \mathbb{T}_{\tau}$; then, $\left\{t_{n_{k}}+\tau\right\} \subset \mathbb{T}$ and $\lim _{k \rightarrow \infty}\left(t_{n_{k}}+\tau\right)=t+\tau$; that is, $t+\tau$ is also right dense. In this case, we have $\sigma(t)=t, \sigma(t+\tau)=$ $t+\tau$. If $\left\{t_{n}\right\}$ has no subsequences contained in $\mathbb{T}_{\tau}$, then, since $\operatorname{dist}\left(\mathbb{T}, \mathbb{T}_{\tau}\right)<\varepsilon$, there exists a sequence $\left\{s_{n}\right\} \subset \mathbb{T}_{\tau}$ such that

$$
\left|s_{n}-t_{n}\right|<\varepsilon, \quad \lim _{n \rightarrow \infty} s_{n}=t .
$$

Hence, $\left\{s_{n}+\tau\right\} \subset \mathbb{T}$ and $\lim _{n \rightarrow \infty}\left(s_{n}+\tau\right)=t+\tau$, that is, $t+\tau$ is right dense. In this case, we also have $\sigma(t)=t, \sigma(t+\tau)=t+\tau$.

On the other hand, if $t$ is right scattered, then there exists an $s(>t) \in \mathbb{T}$ such that $\sigma(t)=s$. If $s+\tau \in \mathbb{T}$, then $\sigma(t+\tau) \leq s+\tau$ and

$$
\sigma(t+\tau)-\sigma(t)-\tau \leq s+\tau-s-\tau=0 .
$$

If $s+\tau \notin \mathbb{T}$, then, since $\operatorname{dist}\left(\mathbb{T}, \mathbb{T}_{\tau}\right)<\varepsilon$, there exists an $s^{*}(>s) \in$ $\mathbb{T}_{\tau}$ such that $s^{*}+\tau \in \mathbb{T}$ and $\left|s-s^{*}\right|<\varepsilon$. Thus, $\sigma(t+\tau) \leq s^{*}+\tau$. Thus,

$$
\sigma(t+\tau)-\sigma(t)-\tau \leq s^{*}+\tau-s-\tau=s^{*}-s .
$$

By the definition of forward jump operator, we know that $\sigma(t+\tau) \geq t+\tau$; then, $\sigma(t+\tau)-\tau \geq t$. Again, by the definition of forward jump operator, we have

$$
\sigma(t) \leq \sigma(t+\tau)-\tau .
$$

Equations (32), (33), and (34) imply that inequality (29) holds. Since $\Pi(\mathbb{T}, \varepsilon)$ is relatively dense, the conclusion of the theorem is true. The proof is complete.

By Theorem 14 and the definitions of the graininess function $\mu(t)$ and $\Pi(\mathbb{T}, \varepsilon)$, we have the following.

Corollary 15. Let $\mathbb{T}$ be an almost periodic time scale; then, for any $\varepsilon>0$, we have that

$$
E\{\mu, \varepsilon\}=\left\{\tau:|\mu(t+\tau)-\mu(t)|<\varepsilon, \forall t \in \mathbb{T}_{\tau}\right\}
$$

is relatively dense.

\section{Almost Periodic Functions on Time Scales}

Let $\mathbb{~ b e}$ an almost time scale; then, we give a definition of almost periodic functions on $\mathbb{T}$.

Definition 16. Let $\mathbb{T}$ be an almost periodic time scale. A function $f \in C\left(\mathbb{T} \times D, \mathbb{E}^{n}\right)$ is called an almost periodic function in $t \in \mathbb{T}$ uniformly for $x \in D$ if the $\varepsilon$-translation set of $f$

$$
\begin{gathered}
E\{\varepsilon, f, S\}=\{\tau \in \Pi(\mathbb{T}, \varepsilon):|f(t+\tau, x)-f(t, x)|<\varepsilon, \\
\left.\forall(t, x) \in \mathbb{\mathbb { T }}_{\tau} \times S\right\}
\end{gathered}
$$

is relatively dense for all $\varepsilon>0$ and for each compact subset $S$ of $D$; that is, for any given $\varepsilon>0$ and each compact subset $S$ of $D$, there exists a constant $l(\varepsilon, S)>0$ such that each interval of length $l(\varepsilon, S)$ contains a $\tau(\varepsilon, S) \in E\{\varepsilon, f, S\} \subset \Pi(\mathbb{T}, \varepsilon)$ such that

$$
|f(t+\tau, x)-f(t, x)|<\varepsilon, \quad \forall(t, x) \in \mathbb{T}_{\tau} \times S .
$$

$\tau$ is called the $\varepsilon$-translation number of $f$ and $l(\varepsilon, S)$ is called the inclusion length of $E\{\varepsilon, f, S\}$. 
In the following, we denote $\widetilde{\mathbb{T}}=\bigcap_{\tau \in \widetilde{\Pi}} \mathbb{\mathbb { T }}_{\tau}$, where $\widetilde{\Pi}=\{\tau \in$ $\left.\mathbb{R}: \mathbb{T}_{\tau} \neq \emptyset\right\}$.

Theorem 17. Let $f \in C\left(\mathbb{T} \times D, \mathbb{E}^{n}\right)$ be almost periodic in $t$ uniformly for $x \in D$; then, it is uniformly continuous and bounded on $\widetilde{\mathbb{T}} \times S$ if $\widetilde{\mathbb{T}} \neq \emptyset$.

Proof. For a given $0<\varepsilon \leq 1$ and some compact set $S \subset D$, there exists a constant $l(\varepsilon, S)$ such that in any interval of length $l(\varepsilon, S)$, there exists $\tau(\varepsilon) \in E\{\varepsilon, f, S\}$ such that

$$
|f(t+\tau, x)-f(t, x)|<\varepsilon \leq 1,
$$

for all $(t, x) \in \widetilde{\mathbb{T}} \times S$. Since $f \in C\left(\mathbb{T} \times D, \mathbb{E}^{n}\right)$, for any $(t, x) \in$ $([0, l(\varepsilon, S)] \cap \mathbb{T}) \times S$, there exists an $M>0$ such that $|f(t, x)|<$ $M$. For any given $t \in \widetilde{\mathbb{T}}$, take $\tau \in E(\varepsilon, f, S) \cap[-t,-t+l(\varepsilon, S)]$. Then, $t+\tau \in[0, l(\varepsilon, S)] \cap \mathbb{T}$. Thus, for $x \in S$, we have

$$
|f(t+\tau, x)|<M, \quad|f(t+\tau, x)-f(t, x)|<1 .
$$

Hence,

$$
|f(t, x)|<|f(t+\tau, x)|+\varepsilon \leq M+1
$$

for all $(t, x) \in \widetilde{\mathbb{T}} \times S$.

Moreover, for any $\varepsilon>0$, let $l_{1}=l_{1}(\varepsilon / 3, S)$ be an inclusion length of $E\{\varepsilon / 3, f, S\}$. We can find a proper point $t_{0}>0$ such that $f(t, x)$ is uniformly continuous on $\left(\left[-t_{0}, t_{0}+l_{1}\right] \cap \mathbb{T}\right) \times S$. Hence, there exists a positive constant $\delta=\delta(\varepsilon / 3, S)<t_{0}$, for any $t_{1}, t_{2} \in\left[-t_{0}, t_{0}+l_{1}\right] \cap \mathbb{T}$ and $\left|t_{1}-t_{2}\right|<\delta$,

$$
\left|f\left(t_{1}, x\right)-f\left(t_{2}, x\right)\right|<\frac{\varepsilon}{3}, \quad \forall x \in S .
$$

Now, we choose arbitrary $v, t \in \widetilde{\mathbb{T}}$, satisfying $|t-v|<\delta$, and we take

$$
\tau \in E\left\{\frac{\varepsilon}{3}, f, S\right\} \cap\left[-t-t_{0},-t+t_{0}+l_{1}\right] .
$$

Then, $t+\tau, v+\tau \in\left[-t_{0}, t_{0}+l_{1}\right] \cap \mathbb{T}$. Thus,

$$
|f(t+\tau, x)-f(v+\tau, x)|<\frac{\varepsilon}{3} \quad \forall x \in S .
$$

Therefore, for $(t, x) \in \widetilde{\mathbb{T}} \times S$, we have

$$
\begin{aligned}
& |f(t, x)-f(v, x)| \\
& \quad \leq|f(t, x)-f(t+\tau, x)|+|f(t+\tau, x)-f(v+\tau, x)| \\
& \quad+|f(v+\tau, x)-f(v, x)|<\varepsilon .
\end{aligned}
$$

So, $f$ is uniformly continuous on $\widetilde{\mathbb{T}} \times S$. The proof is complete.

Remark 18. From Theorem 17, we see that almost periodic functions under Definition 16 may not be bounded on $\mathbb{T}$, so Theorem 3.5 of [12] should assume that $f$ is a bounded function and in the proof of Theorem 3.5 of [12], $D$ should be assumed to be the space formed by all bounded almost periodic functions on an almost periodic time scale $\mathbb{T}$.
For convenience, we introduce the translation operator $T ; T_{\alpha} f(t, x)=g(t, x)$ means that $g(t, x)=\lim _{n \rightarrow+\infty} f(t+$ $\left.\alpha_{n}, x\right)$ and is written only when the limit exists. The mode of convergence, for example, pointwise, uniform, and so forth, will be specified at each use of the symbol.

Theorem 19. Let $f \in C\left(\mathbb{T} \times D, \mathbb{E}^{n}\right)$ be almost periodic in $t$ uniformly for $x \in D$; then, for any given sequence $\alpha^{\prime} \subset \Pi(\mathbb{T}, \varepsilon)$, there exist a subsequence $\beta \subset \alpha^{\prime}$ and $g \in C\left(\widetilde{\mathbb{T}} \times D, \mathbb{E}^{n}\right)$ such that $T_{\beta} f(t, x)=g(t, x)$ holds uniformly on $\widetilde{\mathbb{T}} \times S$ and if $\widetilde{g}(t, x)=$ $g(t, x)$ for $(t, x) \in C\left(\widetilde{\mathbb{T}} \times D, \mathbb{E}^{n}\right)$, then $\widetilde{g}(t, x)$ is almost periodic int uniformly for $x \in D$, where $\widetilde{g} \in C\left(\mathbb{T} \times D, \mathbb{E}^{n}\right)$.

Proof. For any $\varepsilon>0$ and $S \subset D$, let $l=l(\varepsilon / 4, S)$ be an inclusion length of $E\{\varepsilon / 4, f, S\}$. For any given subsequence $\alpha^{\prime}=\left\{\alpha_{n}^{\prime}\right\} \subset \Pi(\mathbb{T}, \varepsilon)$, we denote $\alpha_{n}^{\prime}=\tau_{n}^{\prime}+\gamma_{n}^{\prime}$, where $\tau_{n}^{\prime} \epsilon$ $E\{\varepsilon / 4, f, S\}, \gamma_{n}^{\prime} \in \Pi\left(\mathbb{T}, 2 \varepsilon\right.$ ), and $0 \leq \gamma_{n}^{\prime} \leq l, n=1,2, \ldots$ (in fact, for any interval with length of $l$, there exists $\tau_{n}^{\prime} \in E\{\varepsilon / 4, f, S\}$; thus, we can choose a proper interval with length of $l$ such that $0 \leq \alpha_{n}^{\prime}-\tau_{n}^{\prime} \leq l$; from the definition of $\Pi(\mathbb{T}, \varepsilon)$, it is easy to see that $\left.\gamma_{n}^{\prime}=\alpha_{n}^{\prime}-\tau_{n}^{\prime} \in \Pi(\mathbb{T}, 2 \varepsilon)\right)$. Therefore, there exists a subsequence $\gamma=\left\{\gamma_{n}\right\} \subset \gamma^{\prime}=\left\{\gamma_{n}^{\prime}\right\}$ such that $\gamma_{n} \rightarrow s$ as $n \rightarrow \infty, 0 \leq s \leq l$.

Also, it follows from Theorem 24 that $f(t, x)$ is uniformly continuous on $\mathbb{T} \times S$. Hence, there exists $\delta(\varepsilon, S)>0$ so that $\left|t_{1}-t_{2}\right|<\delta$, for $x \in S$, implies

$$
\left|f\left(t_{1}, x\right)-f\left(t_{2}, x\right)\right|<\frac{\varepsilon}{2} .
$$

Since $\gamma$ is a convergent sequence, there exists $N=N(\delta)$ so that $p, m \geq N$ implies $\left|\gamma_{p}-\gamma_{m}\right|<\delta$. Now, one can take $\alpha \subset \alpha^{\prime}, \tau \subset \tau^{\prime}=\left\{\tau_{n}^{\prime}\right\}$ such that $\alpha, \tau$ common with $\gamma$, then, for any integers $p, m \geq N$ and $t \in \widetilde{\mathbb{T}}$, we have

$$
\begin{aligned}
\mid f( & \left.t+\left(\tau_{p}-\tau_{m}\right), x\right)-f(t, x) \mid \\
\leq & \left|f\left(t+\left(\tau_{p}-\tau_{m}\right), x\right)-f\left(t+\tau_{p}, x\right)\right| \\
& +\left|f\left(t+\tau_{p}, x\right)-f(t, x)\right| \\
< & \frac{\varepsilon}{4}+\frac{\varepsilon}{4}=\frac{\varepsilon}{2} ;
\end{aligned}
$$

that is

$$
\left(\alpha_{p}-\alpha_{m}\right)-\left(\gamma_{p}-\gamma_{m}\right)=\tau_{p}-\tau_{m} \in E\left\{\frac{\varepsilon}{2}, f, S\right\} .
$$

Hence, we can obtain

$$
\begin{aligned}
& \left|f\left(t+\alpha_{p}, x\right)-f\left(t+\alpha_{m}, x\right)\right| \\
& \quad \leq \sup _{(t, x) \in \mathbb{T} \times S}\left|f\left(t+\alpha_{p}, x\right)-f\left(t+\alpha_{m}, x\right)\right| \\
& \quad \leq \sup _{(t, x) \in \mathbb{\pi} \times S}\left|f\left(t+\alpha_{p}-\alpha_{m}, x\right)-f(t, x)\right|
\end{aligned}
$$




$$
\begin{aligned}
& \leq \sup _{(t, x) \in \widetilde{\mathbb{T}} \times S}\left|f\left(t+\alpha_{p}-\alpha_{m}, x\right)-f\left(t+\gamma_{p}-\gamma_{m}, x\right)\right| \\
& \quad+\sup _{(t, x) \in \widetilde{\mathbb{T}} \times S}\left|f\left(t+\gamma_{p}-\gamma_{m}, x\right)-f(t, x)\right| \\
& <\frac{\varepsilon}{2}+\frac{\varepsilon}{2}=\varepsilon .
\end{aligned}
$$

Thus, we can take sequences $\alpha^{(k)}=\left\{\alpha_{n}^{(k)}\right\}, k=1,2, \ldots$, and $\alpha^{(k+1)} \subset \alpha^{(k)} \subset \alpha$ such that, for any integers $m$, $p$, and all $(t, x) \in \widetilde{\mathbb{T}} \times S$, the following holds:

$$
\left|f\left(t+\alpha_{p}^{(k)}, x\right)-f\left(t+\alpha_{m}^{(k)}, x\right)\right|<\frac{1}{k}, \quad k=1,2, \ldots
$$

For all sequences $\alpha^{(k)}, k=1,2, \ldots$, we can take a sequence $\beta=\left\{\beta_{n}\right\}, \beta_{n}=\alpha_{n}^{(n)}$, then it is easy to see that $\left\{f\left(t+\beta_{n}, x\right)\right\} \subset$ $\left\{f\left(t+\alpha_{n}, x\right)\right\}$ for any integers $p, m$ with $p<m$ and all $(t, x) \in$ $\mathbb{T} \times S$, the following holds:

$$
\left|f\left(t+\beta_{p}, x\right)-f\left(t+\beta_{m}, x\right)\right|<\frac{1}{p} .
$$

Therefore, $\left\{f\left(t+\beta_{n}, x\right)\right\}$ converges uniformly on $\widetilde{\mathbb{T}} \times S$; that is, $T_{\beta} f(t, x)=g(t, x)$ holds uniformly on $\widetilde{\mathbb{T}} \times S$, where $\beta=$ $\left\{\beta_{n}\right\} \subset \alpha$.

Next, we will prove that $g(t, x)$ is continuous on $\tilde{\mathbb{\pi}} \times D$. If this is not true, there must exist $\left(t_{0}, x_{0}\right) \in \widetilde{\mathbb{T}} \times D$ such that $g(t, x)$ is not continuous at this point. Then, there exist $\varepsilon_{0}>0$ and sequences $\left\{\delta_{m}\right\},\left\{t_{m}\right\},\left\{x_{m}\right\}$, where $\delta_{m}>0, \delta_{m} \rightarrow 0$ as $m \rightarrow+\infty,\left|t_{0}-t_{m}\right|+\left|x_{0}-x_{m}\right|<\delta_{m}$ and

$$
\left|g\left(t_{0}, x_{0}\right)-g\left(t_{m}, x_{m}\right)\right| \geq \varepsilon_{0} \text {. }
$$

Letting $X=\left\{x_{m}\right\} \bigcup\left\{x_{0}\right\}$, obviously, $X$ is a compact subset of $D$. Hence, there exists positive integer $N=N\left(\varepsilon_{0}, X\right)$ so that $n>N$ implies

$$
\begin{gathered}
\left|f\left(t_{m}+\beta_{n}, x_{m}\right)-g\left(t_{m}, x_{m}\right)\right|<\frac{\varepsilon_{0}}{3} \quad \forall m \in \mathbb{Z}^{+}, \\
\left|f\left(t_{0}+\beta_{n}, x_{0}\right)-g\left(t_{0}, x_{0}\right)\right|<\frac{\varepsilon_{0}}{3} .
\end{gathered}
$$

According to the uniform continuity of $f(t, x)$ on $\widetilde{\mathbb{T}} \times X$, for sufficiently large $m$, we have

$$
\left|f\left(t_{0}+\beta_{n}, x_{0}\right)-f\left(t_{m}+\beta_{n}, x_{m}\right)\right|<\frac{\varepsilon_{0}}{3} .
$$

From (52)-(53), we get

$$
\left|g\left(t_{0}, x_{0}\right)-g\left(t_{m}, x_{m}\right)\right|<\varepsilon_{0}
$$

this contradicts (51). Therefore, $g(t, x)$ is continuous on $\widetilde{\mathbb{T}} \times D$.

Finally, for any compact set $S \subset D$ and given $\varepsilon>0$, one can take $\tau \in E\{\varepsilon, f, S\}$, then for all $(t, x) \in \widetilde{\mathbb{T}} \times S$, the following holds:

$$
\left|f\left(t+\left(\beta_{n}+\tau\right), x\right)-f\left(t+\beta_{n}, x\right)\right|<\varepsilon .
$$

Let $n \rightarrow+\infty$, for all $(t, x) \in \widetilde{\mathbb{T}} \times S$, we have

$$
|g(t+\tau, x)-g(t, x)| \leq \varepsilon,
$$

which implies that $E\{\varepsilon, g, S\}$ is relatively dense. Therefore, $\tilde{g}(t, x)$ is almost periodic in $t$ uniformly for $x \in D$. This completes the proof.

Theorem 20. Let $f(t, x) \in C\left(\mathbb{T} \times D, \mathbb{E}^{n}\right)$, if for any sequence $\alpha^{\prime} \subset \Pi(\mathbb{T}, \varepsilon)$, there exists $\alpha \subset \alpha^{\prime}$ such that $T_{\alpha} f(t, x)$ exists uniformly on $\widetilde{\mathbb{T}} \times S$, then $f(t, x)$ is almost periodic in $t$ uniformly for $x \in D$.

Proof. For contradiction, if this is not true, then there exist $\varepsilon_{0}>0$ and $S \subset D$ such that for any sufficiently large $l>0$, we can find an interval with length of $l$ and there is no $\varepsilon_{0^{-}}$ translation numbers of $f(t, x)$ in this interval, that is, every point in this interval is not in $E\left\{\varepsilon_{0}, f, S\right\}$.

One can take a number $\alpha_{1}^{\prime} \in \Pi(\mathbb{T}, \varepsilon)$ and find an interval $\left(a_{1}, b_{1}\right)$ with $b_{1}-a_{1}>2\left|\alpha_{1}^{\prime}\right|$, where $a_{1}, b_{1} \in \Pi$ such that there are no $\varepsilon_{0}$-translation numbers of $f(t, x)$ in this interval. Next, taking $\alpha_{2}^{\prime}=(1 / 2)\left(a_{1}+b_{1}\right)$, obviously, $\alpha_{2}^{\prime}-\alpha_{1}^{\prime} \in\left(a_{1}, b_{1}\right)$, so $\alpha_{2}^{\prime}-\alpha_{1}^{\prime} \notin E\left\{\varepsilon_{0}, f, S\right\}$; then, one can find an interval $\left(a_{2}, b_{2}\right)$ with $b_{2}-a_{2}>2\left(\left|\alpha_{1}^{\prime}\right|+\left|\alpha_{2}^{\prime}\right|\right)$, where $a_{2}, b_{2} \in \Pi(\mathbb{T}, \varepsilon)$ such that there is no $\varepsilon_{0}$-translation numbers of $f(t, x)$ in this interval. Next, taking $\alpha_{3}^{\prime}=(1 / 2)\left(a_{2}+b_{2}\right)$, obviously, $\alpha_{3}^{\prime}-\alpha_{2}^{\prime}, \alpha_{3}^{\prime}-\alpha_{1}^{\prime} \notin$ $E\left\{\varepsilon_{0}, f, S\right\}$. One can repeat these processes again and again, one can find $\alpha_{4}^{\prime}, \alpha_{5}^{\prime}, \ldots$, such that $\alpha_{i}^{\prime}-\alpha_{j}^{\prime} \notin E\left\{\varepsilon_{0}, f, S\right\}, i>j$. Hence, for any $i \neq j, i, j=1,2, \ldots$, without loss of generality, lettting $i>j$, for $x \in S$ we have

$$
\begin{aligned}
& \sup _{(t, x) \in \widetilde{\mathbb{T}} \times S}\left|f\left(t+\alpha_{i}^{\prime}, x\right)-f\left(t+\alpha_{j}^{\prime}, x\right)\right| \\
& =\sup _{(t, x) \in \widetilde{\mathbb{T}} \times S}\left|f\left(t+\left(\alpha_{i}^{\prime}-\alpha_{j}^{\prime}\right), x\right)-f(t, x)\right| \geq \varepsilon_{0} .
\end{aligned}
$$

Therefore, there is no uniformly convergent subsequence of $\left\{f\left(t+\alpha_{n}^{\prime}, x\right)\right\}$ for $(t, x) \in \widetilde{\mathbb{T}} \times S$; this is a contradiction. Thus, $f(t, x)$ is almost periodic in $t$ uniformly for $x \in D$. This completes the proof.

Based on Theorems 19 and 20, and Remark 18, we give the following definition of uniformly almost periodic functions.

Definition 21. Let $f(t, x) \in C\left(\mathbb{T} \times D, \mathbb{E}^{n}\right)$, if for any given sequence $\alpha^{\prime} \subset \Pi(\mathbb{T}, \varepsilon)$, there exists a subsequence $\alpha \subset \alpha^{\prime}$ such that $T_{\alpha} f(t, x)$ exists uniformly on $\widetilde{\mathbb{T}} \times S$, then $f(t, x)$ is called an almost periodic function in $t$ uniformly for $x \in D$.

Definition 22. Let $\mathbb{T}$ be an almost periodic time scale. For any $t \in \mathbb{T}, \tau \in \Pi(\mathbb{T}, \varepsilon)$, we define

$$
t \tilde{+} \tau= \begin{cases}t+\tau & \text { if } t+\tau \in \mathbb{T}, \\ t^{*}+\tau & \text { if } t+\tau \notin \mathbb{T},\end{cases}
$$

where $t^{*} \in \mathbb{\mathbb { T }}_{\tau}$ satisfies that $\operatorname{dist}\left(t, \mathbb{\mathbb { T }}_{\tau}\right)=\left|t-t^{*}\right|<\varepsilon$ and $\left(t-t^{*}\right) \operatorname{sign}(\tau)>0$.

Based on Definition 22, we give a definition of almost periodic functions on time scales. 
Definition 23. Let $\mathbb{\square}$ be an almost periodic time scale. A function $f \in C\left(\mathbb{T} \times D, \mathbb{E}^{n}\right)$ is called an almost periodic function in $t \in \mathbb{T}$ uniformly for $x \in D$ if the $\varepsilon$-translation set of $f$

$$
\begin{gathered}
E\{\varepsilon, f, S\}=\{\tau \in \Pi(\mathbb{T}, \varepsilon):|f(t \tilde{+} \tau, x)-f(t, x)|<\varepsilon, \\
\forall(t, x) \in \mathbb{T} \times S\}
\end{gathered}
$$

is relatively dense for all $\varepsilon>0$ and for each compact subset $S$ of $D$; that is, for any given $\varepsilon>0$ and each compact subset $S$ of $D$, there exists a constant $l(\varepsilon, S)>0$ such that each interval of length $l(\varepsilon, S)$ contains a $\tau(\varepsilon, S) \in E\{\varepsilon, f, S\}$ such that

$$
|f(t \tilde{+} \tau, x)-f(t, x)|<\varepsilon, \quad \forall(t, x) \in \mathbb{T} \times S .
$$

$\tau$ is called the $\varepsilon$-translation number of $f$ and and $l(\varepsilon, S)$ is called the inclusion length of $E\{\varepsilon, f, S\}$.

Under sense of Definition 23, we have the following.

Theorem 24. Let $f \in C\left(\mathbb{T} \times D, \mathbb{E}^{n}\right)$ be almost periodic in $t$ uniformly for $x \in D$; then, for any $\varepsilon>0$, it is uniformly continuous and bounded on $\mathbb{T} \times S$.

Proof. For a given $\varepsilon \leq 1$ and some compact set $S \subset D$, there exists a constant $l(\varepsilon, S)$ such that in any interval of length $l(\varepsilon, S)$, there exists $\tau(\varepsilon) \in E\{\varepsilon, f, S\}$ such that

$$
|f(t \tilde{+} \tau, x)-f(t, x)|<\varepsilon \leq 1, \quad \forall(t, x) \in \mathbb{T} \times S .
$$

Since $f \in C\left(\mathbb{T} \times D, \mathbb{R}^{n}\right)$, for any $(t, x) \in([-1-\bar{\mu}, l(\varepsilon, S)+1+\bar{\mu}] \cap$ $\mathbb{T}) \times S$, where $\mu=\sup _{t \in \mathbb{T}} \mu(t)$, there exists an $M>0$ such that $|f(t, x)|<M$. For any given $t \in \mathbb{T}$, take $\tau \in E(\varepsilon, f, S) \cap[-t-$ $1-\bar{\mu},-t+1+\bar{\mu}+l(\varepsilon, S)]$. Then, $t \tilde{+} \tau \in[-1-\bar{\mu}, l(\varepsilon, S)+1+\bar{\mu}] \cap \mathbb{T}$; thus, for $(t, x) \in \mathbb{T} \times S$, we have

$$
|f(t \tilde{+} \tau, x)|<M, \quad|f(t \tilde{+} \tau, x)-f(t, x)|<1 .
$$

Therefore, for all $(t, x) \in \mathbb{T} \times S$, we have $|f(t, x)|<M+1$.

Moreover, for any $\varepsilon>0$, let $l_{1}=l_{1}(\varepsilon / 3, S)$ be an inclusion length of $E(\varepsilon / 3, f, S)$. We can find a proper point $t_{0}>0$. Since $f(t, x)$ is uniformly continuous on $\left(\left[-t_{0}-1-\bar{\mu}, t_{0}+l_{1}+1+\right.\right.$ $\bar{\mu}] \cap \mathbb{T}) \times S$, there exists a positive constant $\delta=\delta(\varepsilon / 3, S)<$ $\min \left\{\bar{\mu}+1, t_{0}\right\}$, for any $t_{1}, t_{2} \in\left[-t_{0}-1-\bar{\mu}, t_{0}+l_{1}+1+\bar{\mu}\right] \cap \mathbb{\square}$ and $\left|t_{1}-t_{2}\right|<\delta$,

$$
\left|f\left(t_{1}, x\right)-f\left(t_{2}, x\right)\right|<\frac{\varepsilon}{3} \quad \forall x \in S .
$$

Choose arbitrary $v, t \in \mathbb{T}$, satisfying $|t-v|<\delta$, and take

$$
\tau \in E\left(\frac{\varepsilon}{3}, f\right) \cap\left[-t-t_{0}-1-\bar{\mu},-t+t_{0}+1+\bar{\mu}+l_{1}\right]
$$

then, $t \tilde{+} \tau, v \tilde{+} \tau \in\left[-t_{0}-1-\bar{\mu}, t_{0}+l_{1}+1+\bar{\mu}\right] \cap \mathbb{T}$, so

$$
|f(t \tilde{+} \tau, x)-f(v \tilde{+} \tau, x)|<\frac{\varepsilon}{3} \quad \forall x \in S .
$$

Therefore, for $(t, x) \in \mathbb{T} \times S$, we have

$$
\begin{aligned}
& |f(t, x)-f(v, x)| \\
& \leq|f(t, x)-f(t \tilde{+} \tau, x)|+|f(t \tilde{+} \tau, x)-f(v \tilde{+} \tau, x)| \\
& \quad+|f(v \widetilde{+} \tau, x)-f(v, x)|<\varepsilon .
\end{aligned}
$$

The proof is complete.
Finally, we give a very general definition of almost periodic time scales that can support almost periodic functions defined by Definitions 16, 21, and 22 as follows.

Definition 25. A time scale $\mathbb{T}$ is called an almost periodic time scale if

$$
\Pi:=\left\{\tau \in \mathbb{R}: \mathbb{T}_{\tau} \neq \emptyset\right\}
$$

is relatively dense in $\mathbb{R}$, where $\mathbb{T}_{\tau}=\mathbb{T} \cap\{\mathbb{T}-\tau\}$ or $\mathbb{\mathbb { V }}_{\tau}=\mathbb{\mathbb { T }} \cap$ $\{\mathbb{T} \pm \tau\}$.

Remark 26. Obviously, if $\mathbb{T}$ is an almost periodic time scale, then inf $\mathbb{T}=-\infty$ and sup $\mathbb{T}=+\infty$. If $\mathbb{T}$ is an almost periodic time scale under Definitions 2 and 9 , then $\mathbb{T}$ is also an almost periodic time scale under Definition 25, but not vice versa. For example, $\mathbb{T}=\mathbb{Z} \cup\{1 / 3,1 / 5\}$ is an almost periodic time scale under Definition 25, but it is not an almost periodic time scale under Definitions 2 and 9.

Remark 27. In this paper, we have proposed several new types of almost periodic time scales and almost periodic functions on time scales, but we can only establish a few of their properties. In order to study the almost periodic solutions of dynamic equations, we still have many problems to be solved. For example, we need to establish the hull theory of almost periodic functions and the theory of exponential dichotomy to linear dynamic equations on those new types of almost periodic time scales and so on.

\section{Conflict of Interests}

The authors declare that there is no conflict of interests regarding the publication of this paper.

\section{Acknowledgments}

The authors are deeply grateful to the reviewers for their careful reading of this paper and helpful comments, which have been very useful for improving the quality of this paper. This work is supported by the National Natural Science Foundation of China under Grant 11361072.

\section{References}

[1] H. Bohr, "Zur theorie der fast periodischen funktionen," Acta Mathematica, vol. 45, no. 1, pp. 29-127, 1925.

[2] S. Hilger, "Analysis on measure chains-a unified approach to continuous and discrete calculus," Results in Mathematics, vol. 18, no. 1-2, pp. 18-56, 1990.

[3] Y. K. Li and C. Wang, "Uniformly almost periodic functions and almost periodic solutions to dynamic equations on time scales," Abstract and Applied Analysis, vol. 2011, Article ID 341520, 22 pages, 2011.

[4] Y. Li and C. Wang, "Pseudo almost periodic functions and pseudo almost periodic solutions to dynamic equations on time scales," Advances in Difference Equations, vol. 2012, article 77, 2012.

[5] C. Lizama and J. G. Mesquita, "Almost automorphic solutions of dynamic equations on time scales," Journal of Functional Analysis, vol. 265, no. 10, pp. 2267-2311, 2013. 
[6] C. Wang and Y. Li, "Weighted pseudo almost automorphic functions with applications to abstract dynamic equations on time scales," Annales Polonici Mathematici, vol. 108, no. 3, pp. 225-240, 2013.

[7] C. Wang and R. P. Agarwal, "Weighted piecewise pseudo almost automorphic functions with applications to abstract impulsive $\nabla$-dynamic equations on time scales," Advances in Difference Equations, vol. 2014, article 153, 2014.

[8] C. Lizama and J. G. Mesquita, "Almost automorphic solutions of non-autonomous difference equations," Journal of Mathematical Analysis and Applications, vol. 407, no. 2, pp. 339-349, 2013.

[9] J. Gao, Q.-R. Wang, and L.-W. Zhang, "Existence and stability of almost-periodic solutions for cellular neural networks with time-varying delays in leakage terms on time scales," Applied Mathematics and Computation, vol. 237, pp. 639-649, 2014.

[10] Y. Li and L. Yang, "Almost automorphic solution for neutral type high-order Hopfield neural networks with delays in leakage terms on time scales," Applied Mathematics and Computation, vol. 242, pp. 679-693, 2014.

[11] C. Lizama, J. G. Mesquita, and R. Ponce, "A connection between almost periodic functions defined on timescales and R," Applicable Analysis, vol. 93, no. 12, pp. 2547-2558, 2014.

[12] C. Wang and R. P. Agarwal, "A further study of almost periodic time scales with some notes and applications," Abstract and Applied Analysis, vol. 2014, Article ID 267384, 11 pages, 2014.

[13] M. Bohner and A. Peterson, Dynamic Equations on Time Scales: An Introduction with Applications, Birkhäuser, Boston, Mass, USA, 2001.

[14] A. M. Fink, Almost Periodic Differential Equations, Springer, Berlin, Germany, 1974.

[15] E. R. Kaufmann and Y. N. Raffoul, "Periodic solutions for a neutral nonlinear dynamical equation on a time scale," Journal of Mathematical Analysis and Applications, vol. 319, no. 1, pp. 315-325, 2006. 


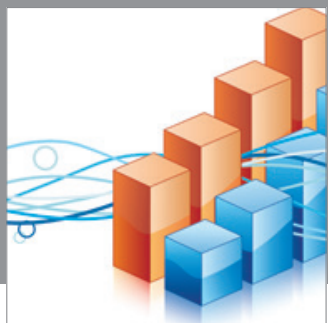

Advances in

Operations Research

mansans

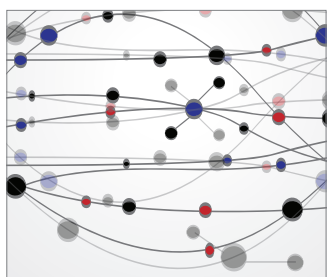

The Scientific World Journal
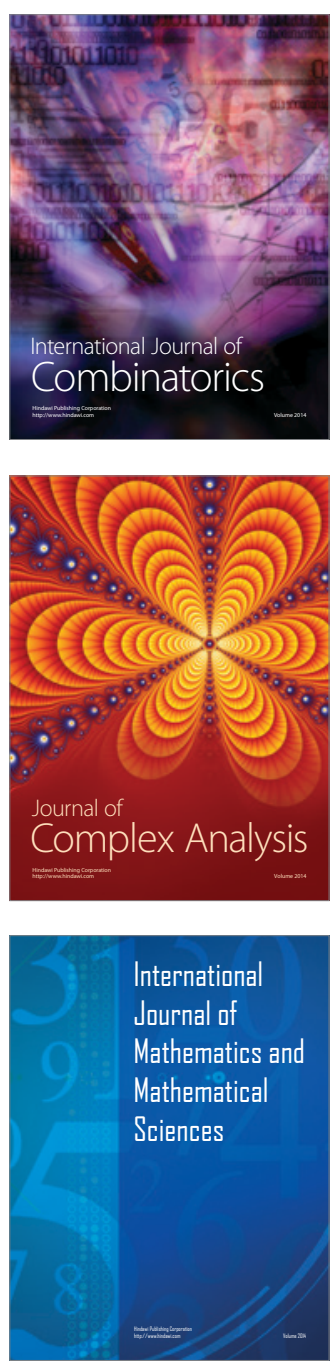
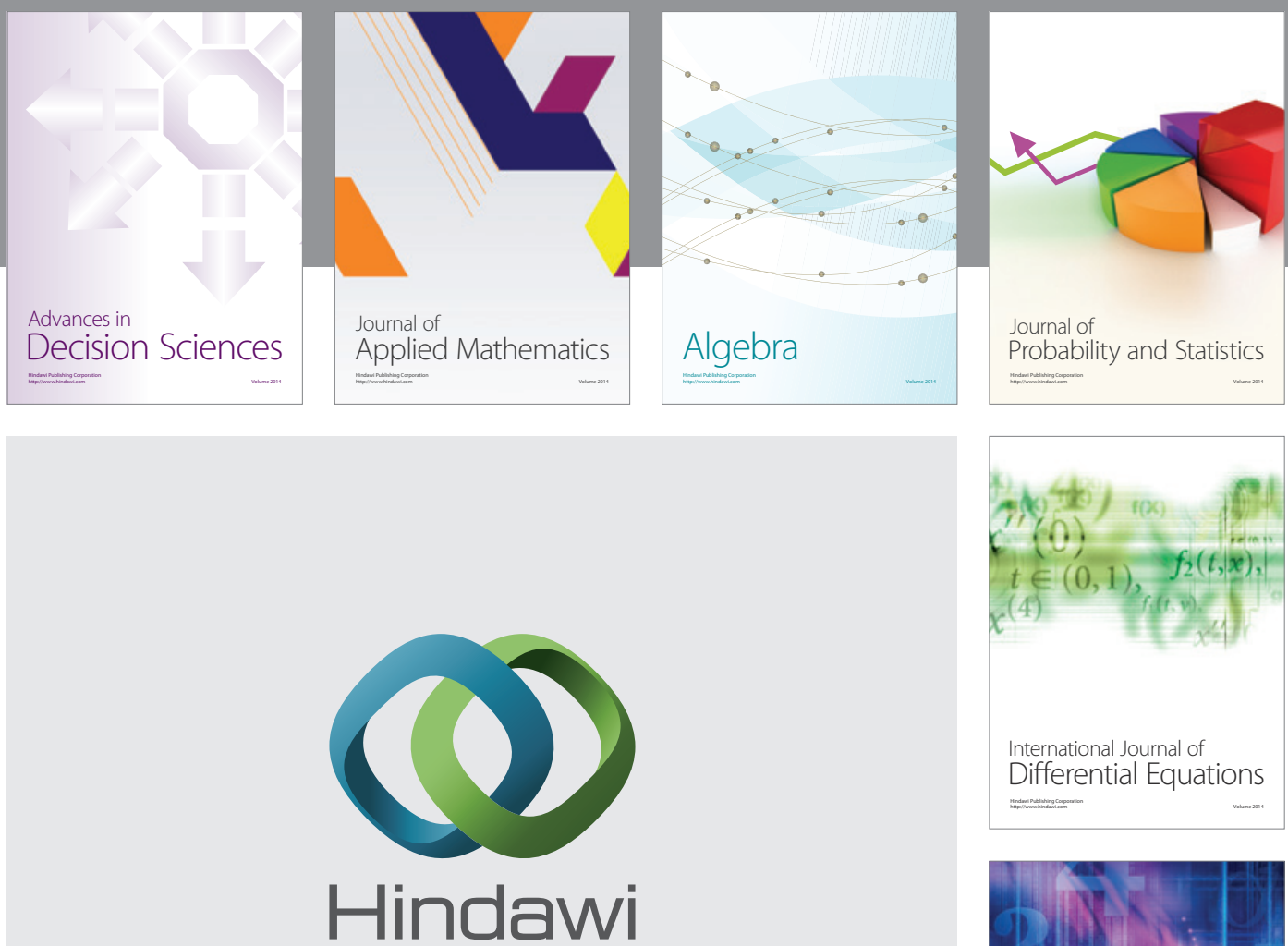

Submit your manuscripts at http://www.hindawi.com
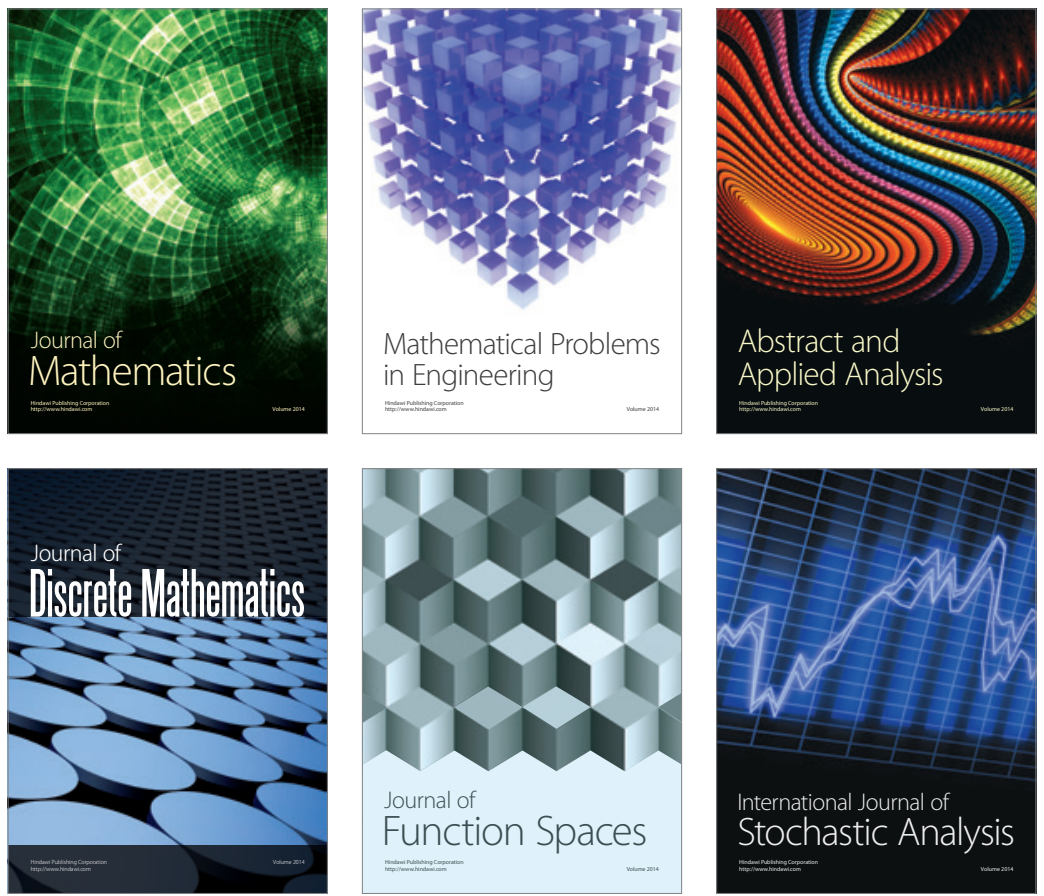

Journal of

Function Spaces

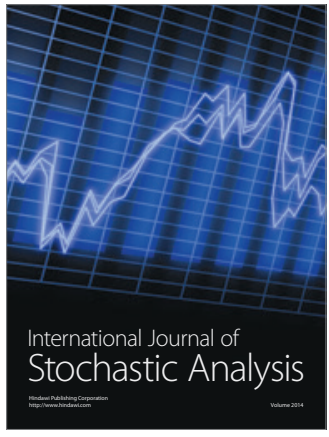

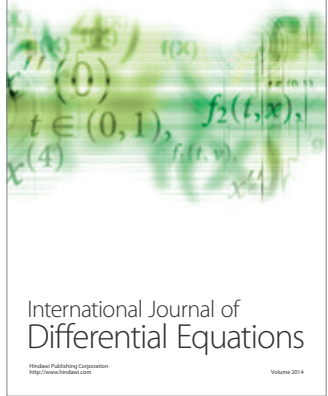
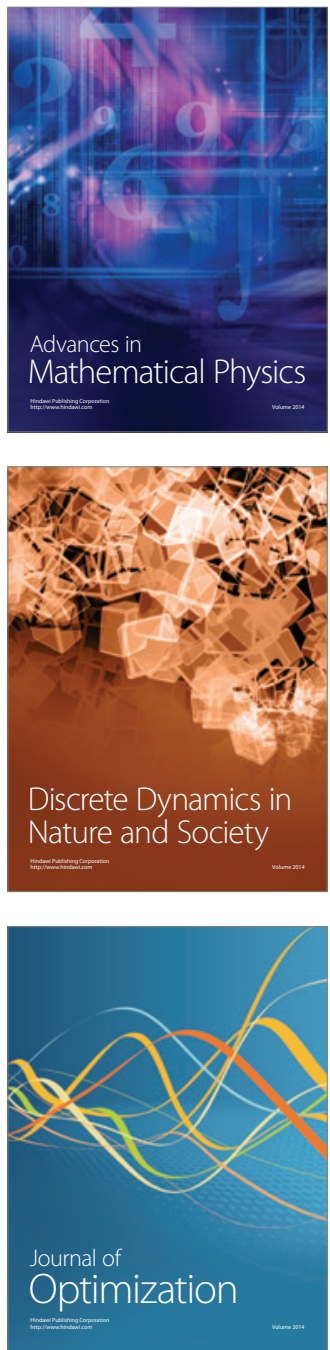